\title{
ÁCIDO GIBERÉLICO ISOLADO OU ASSOCIADO COM CERA NA CONSERVAÇÃO PÓS-COLHEITA DA LIMA ÁCIDA "TAHITI"
}

\author{
GIBBERELIC ACID ALONE OR ASSOCIATED WITH WAX IN \\ THE POST-HARVEST OF “TAHITI" LIME
}

\author{
Luiz Antonio BIASI ${ }^{1} \Xi^{\circ}$ \\ Flávio ZANETTE²
}

\begin{abstract}
RESUMO
A manutenção da coloração verde da casca da lima ácida 'Tahiti' é importante para garantir o valor comercial dos frutos, sendo possível prolongar sua vida por meio de produtos que reduzem a degradação da clorofila, como a cera e o ácido giberélico $\left(\mathrm{GA}_{3}\right)$. O objetivo deste trabalho foi estudar o efeito destes produtos sobre a maturação dos frutos, visando ao aumento do período de conservação pós-colheita. Este experimento foi realizado com frutos coletados de um pomar comercial em Marília, SP, e instalado no dia 07 de novembro de 1998, em Curitiba, PR. Os tratamentos foram os seguintes: 1) Testemunha; 2) $\left.\left.\left.\mathrm{GA}_{3}\left(100 \mathrm{mg} \cdot \mathrm{L}^{-1}\right) ; 3\right) \mathrm{GA}_{3}\left(200 \mathrm{mg} \cdot \mathrm{L}^{-1}\right) ; 4\right) \mathrm{GA}_{3}\left(400 \mathrm{mg} \cdot \mathrm{L}^{-1}\right) ; 5\right) \mathrm{GA}_{3}\left(100 \mathrm{mg} \cdot \mathrm{L}^{-1}\right)+$ cera; 6) $\mathrm{GA}_{3}\left(200 \mathrm{mg} \cdot \mathrm{L}^{-1}\right)+$ cera; 7$) \mathrm{GA}_{3}\left(400 \mathrm{mg} \cdot \mathrm{L}^{-1}\right)+$ cera. O delineamento experimental foi o inteiramente casualizado, com três repetições e 16 frutos por parcela. A aplicação do $\mathrm{GA}_{3}$ foi realizada pela imersão dos frutos nas soluções por dois minutos e a cera foi aplicada após seis horas. Os frutos de cada parcela foram acondicionados em sacos de polietileno com 16 furos e mantidos a $7^{\circ} \mathrm{C}$. As avaliações foram realizadas em intervalos de 15 dias. A aplicação de $\mathrm{GA}_{3}$ e cera não influenciaram as características químicas do suco $(\mathrm{pH}$, teor de sólidos solúveis e acidez). Houve redução da acidez e aumento no $\mathrm{pH}$ do suco durante o armazenamento, em todos os tratamentos. $\mathrm{A}$ aplicação de $\mathrm{GA}_{3}$ foi eficiente na manutenção da coloração verde da casca dos frutos. Todas as concentrações testadas foram superiores à testemunha e não diferiram entre si, nas avaliações realizadas aos 30 e 60 dias, quanto ao teor de clorofila total. A tendência de degradação da clorofila foi semelhante, em todos os tratamentos que apresentaram regressões quadráticas significativas. Nos primeiros 15 dias de armazenamento, a degradação atingiu cerca de 50\%. Aos 30 dias, a degradação foi cerca de $33 \%$ menos acentuada para os tratamentos com $\mathrm{GA}_{3}$, enquanto na testemunha a perda foi de $57 \%$. Quanto à relação entre clorofila $a / b$, houve tendência de aumento na relação aos 30 dias, com posterior redução. Conclui-se que o $\mathrm{GA}_{3}$, em concentrações de 100 a 400 $\mathrm{mg} \cdot \mathrm{L}^{-1}$, é eficiente na manutenção da coloração verde da casca da lima ácida 'Tahiti', independente da aplicação de cera.
\end{abstract}

Palavras-chave: Citrus latifolia, ácido giberélico, cera, clorofila.

\begin{abstract}
The colour conservation of the peel green of the lime 'Tahiti' is very important for its commercial value and it's possible by applying products that avoid chlorophyll degradation. Aiming at studying these products effects in fruit rippening, to increased the fruit conservation, this work was carried out with fruit collected in a orchard located in Marília, SP, and installed at 07/11/1998 in Curitiba, PR. The treatments were the following: 1) control; 2) $\left.\left.\mathrm{GA}_{3}\left(100 \mathrm{mg} \cdot \mathrm{L}^{-1}\right) ; 3\right) \mathrm{GA}_{3}\left(200 \mathrm{mg} \cdot \mathrm{L}^{-1}\right) ; 4\right) \mathrm{GA}_{3}(400 \mathrm{mg} \cdot \mathrm{L}-$ 1); 5) $\mathrm{GA}_{3}\left(100 \mathrm{mg} \cdot \mathrm{L}^{-1}\right)+$ wax; 6) $\mathrm{GA}_{3}\left(200 \mathrm{mg}^{\mathrm{L} \mathrm{L}^{-1}}\right)+$ wax; 7$) \mathrm{GA}_{3}\left(400 \mathrm{mg} \cdot \mathrm{L}^{-1}\right)+$ wax. The experimental design was completely randomized with three replicates and 16 fruits per plot. The $\mathrm{GA}_{3}$ was applied by immersion for two minutes in the solutions and the wax was applicated six hours after. The fruits were stored in polyethylene bags with 16 perforations at $7^{\circ} \mathrm{C}$. The evaluations were maded each 15
\end{abstract}

\footnotetext{
1 Engenheiro Agrônomo, Dr., Professor Adjunto, Departamento de Fitotecnia e Fitossanitarismo, Setor de Ciências Agrárias, UFPR. Caixa Postal 19061. 80001-970. Curitiba-PR. E-mail: labiasi@agrarias.ufpr.br. Bolsista do CNPq. $\risingdotseq$ Autor para correspondência.

2 Engenheiro Agrônomo, Dr., Professor Titular, Departamento de Fitotecnia e Fitossanitarismo, Setor de Ciências Agrárias, UFPR.
} 
BIASI, L. A.; ZANETTE, F. Ácido Giberélico Isolado...

days. The $\mathrm{GA}_{3}$ and wax application had no effect in the juice biochemistry characteristics $(\mathrm{pH}$, soluble solids and acidity). There was an acidity decrease and a pH increase during the storage period in all treatments. The $\mathrm{GA}_{3}$ application was efficient to delay the degreening. All the concentrations of $\mathrm{GA}_{3}$ were higher than control and didn't differ among one another in relation to the total chlorophyll. The degradation of chlorophyll showed the same tendency in all treatments with quadratic comportment. The degradation was $50 \%$ in the first 15 days. The fruits treated with $\mathrm{GA}_{3}$ showed about $33 \%$ of chlorophyll degradation at 30 days, while the control lost $57 \%$. The relation of chlorophyll a/b increased until 30 days and decreased after that period of time. The conclusion is that $\mathrm{GA}_{3}$ at 100 until 400 $\mathrm{mg} . \mathrm{L}^{-1}$ is efficient to avoid the degreening of lime 'Tahiti' independent of wax application.

Key-words: Citrus latifolia, gibberelic acid, wax, chlorophyll.

\section{INTRODUÇÃO}

A lima ácida 'Tahiti', também conhecida como limão 'Tahiti', é uma das espécies cítricas de maior importância comercial, com área cultivada de aproximadamente 30.000 ha (5), sendo os seus frutos destinados para o consumo ao natural ou na forma de suco concentrado, tanto para o mercado interno quanto externo (8). A colheita dos frutos é realizada quando eles ainda estão verdes. A produção é concentrada no primeiro semestre do ano, época de menor preço para o fruto (6). Para prolongar a vida comercial do produto e obter melhores cotações, diversas técnicas podem ser utilizadas, como armazenamento refrigerado, com tempera-turas de $8^{\circ} \mathrm{C}(6,9)$, uso de embalagens de polietileno $(3,9,13)$, aplicação de cera $(9,14)$ e aplicação de reguladores de crescimento $(2,9,12)$.

A manutenção da coloração verde da casca da lima ácida 'Tahiti' é fundamental para garantir a qualidade dos frutos e, conseqüentemente, sua comercialização. Durante a maturação dos frutos, é natural que ocorra degradação da clorofila e aparecimento de pigmentos amarelos (carotenóides) (4). Entre os reguladores de crescimento envolvidos na maturação, as giberelinas são conhecidas por atrasar o surgimento da coloração amarelada na casca dos frutos cítricos (11). Este efeito foi observado por Barros et al. (2) em experiência realizada com a lima ácida 'Tahiti', em que o tratamento dos frutos com $40 \mathrm{mg} . \mathrm{L}^{-1}$ de ácido giberélico $\left(\mathrm{GA}_{3}\right)$ promoveu atraso no amarelecimento.

Este experimento pretendeu verificar o efeito do $\mathrm{GA}_{3}$ isolado e em combinação com cera para retardar a maturação da lima ácida 'Tahiti' durante o armazenamento refrigerado.

\section{METODOLOGIA}

O experimento foi instalado nas dependências do Departamento de Fitotecnia e Fitossanitarismo do Setor de Ciências Agrárias da Universidade Federal do Paraná, em Curitiba, PR.

Os frutos da lima ácida 'Tahiti' foram coletados de um pomar comercial em Marília, SP. A seleção eliminou aqueles com manchas claras na casca, presença de lesões mecânicas ou fúngicas e com massa inferior a $60 \mathrm{~g}$ ou superior a $90 \mathrm{~g}$. O experimento foi instalado no dia sete de novembro de 1998. Os tratamentos foram os seguintes: 1) Testemunha; 2) $\mathrm{GA}_{3}\left(100 \mathrm{mg} \cdot \mathrm{L}^{-1}\right)+$ cera; 3) $\mathrm{GA}_{3}\left(200 \mathrm{mg} \cdot \mathrm{L}^{-1}\right)+$ cera; 4$) \mathrm{GA}_{3}\left(400 \mathrm{mg} \cdot \mathrm{L}^{-}\right.$ $\left.{ }^{1}\right)+$ cera; 5) $\mathrm{GA}_{3}\left(100 \mathrm{mg}^{-\mathrm{L}^{-1}}\right)$; 6) $\mathrm{GA}_{3}\left(200 \mathrm{mg} \cdot \mathrm{L}^{-1}\right)$; e 7) $\mathrm{GA}_{3}\left(400 \mathrm{mg} \cdot \mathrm{L}^{-1}\right)$.

O delineamento experimental utilizado foi o inteiramente casualizado, com três repetições. Cada parcela foi composta por 16 frutos.

A aplicação do $\mathrm{GA}_{3}$ foi realizada pela imersão dos frutos nas respectivas soluções por um período de 2 min. Após a aplicação, os frutos permaneceram em local ventilado e sombreado, por $6 \mathrm{~h}$, até ficarem completamente secos. Nos tratamentos com aplicação de cera, esta foi aplicada por meio de pulverização, após o período de secagem. Para a secagem da cera foi considerado o período de $1 \mathrm{~h}$. Os frutos completamente secos foram acondicionados em sacos de polietileno transparente $(25 \mathrm{~cm} \times 40 \mathrm{~cm})$. Em cada um foram colocados os 16 frutos que compunham cada parcela. Em seguida, os sacos foram mantidos sob refrigeração à temperatura de $7^{\circ} \mathrm{C}$ e umidade relativa de $85 \%$.

As avaliações foram realizadas em intervalos de 15 dias, sendo uma avaliação inicial, na instalação do experimento, e quatro avaliações posteriores, até 60 dias após a instalação. Em cada avaliação foram utilizados quatro frutos de cada parcela. Os parâmetros analisados foram: $\mathrm{pH}$, acidez e teor de sólidos solúveis do suco e quantidade de clorofila $a, b$ e total presentes na casca.

O teor de sólidos solúveis foi determinado por meio de refratômetro de mão, utilizando duas gotas da mistura do suco puro dos quatro frutos de cada parcela. $\mathrm{O} \mathrm{pH}$ foi determinado pela leitura direta do suco dos quatro frutos diluídos em água deionizada na proporção de $10 \mathrm{ml}$ de suco para $90 \mathrm{ml}$ de água, num potenciômetro. A acidez foi determinada por meio da titulação do suco diluído, com $\mathrm{NaOH} 1 \mathrm{~N}$ até atingir o $\mathrm{pH} 8,1$. As quantidades de clorofila $a, b$ e total foram determinadas utilizando a metodologia de Arnon (1) modificada por Manfroi et al. (12) e adaptada neste trabalho. De cada fruto analisado foram retirados $10 \mathrm{~cm}^{2} \mathrm{da}$ 
casca da região equatorial do fruto, na forma de uma tira de $1 \mathrm{~cm}$ de largura e $10 \mathrm{~cm}$ de comprimento. As cascas dos quatro frutos de cada parcela foram colocadas em frascos de vidro hermeticamente fechados, contendo $20 \mathrm{ml}$ de solução extratora de acetona $80 \%$. Os frascos foram revestidos com papel alumínio e mantidos sob refrigeração por $72 \mathrm{~h}$, quando procedeu-se a leitura da absorbância da solução num espectrofotômetro, nos comprimentos de onda de 645 e 663 nm. Os teores de clorofila $a, b$ e total foram obtidos pelas seguintes fórmulas:

\section{Clorofila a: $\quad$ 12,7(Absorbância 663nm) - 2,7(Absorbância 645nm) \\ Clorofila b: $\quad$ 22,9(Absorbância 645nm) - 4,7(Absorbância 663nm) \\ Clorofila total: 8,0(Absorbância 663nm) + 20,2(Absorbância 645nm)}

Todos os dados obtidos foram submetidos à análise de variância, sendo utilizada a análise de regressão polinomial para o efeito do tempo de armazenamento e o teste de Tukey para a compara- ção das médias dos tratamentos. As análises foram realizadas pelo programa estatístico SANEST.

\section{RESULTADOS E DISCUSSÃO}

A aplicação de $\mathrm{GA}_{3}$ e de cera não influenciaram as características químicas do suco dos frutos. Quanto ao teor de sólidos solúveis, foram verificadas pequenas diferenças entre os tratamentos, nas avaliações de 15, 30 e 60 dias (Tabela 1), mas não demonstraram tendência clara de estarem relacionadas com o efeito da aplicação dos produtos, sendo assim atribuídas ao acaso. Os valores do teor de sólidos solúveis observados neste trabalho foram muito próximos aos encontrados por Manfroi et al. (12) e Carvalho et al. (3), que também trabalharam com a conservação pós-colheita da lima ácida 'Tahiti'.

$\mathrm{O} \mathrm{pH}$ variou muito pouco entre os tratamentos, sendo verificada diferença significativa apenas aos 30 dias (Tabela 2), o que não caracteriza efeito próprio do tratamento, mas sim pequena variação casual.

TABELA 1 -Efeito da aplicação de ácido giberélico $\left(\mathrm{GA}_{3}\right)$ isolado ou associado com cera no teor de sólidos solúveis do suco, durante o armazenamento refrigerado da lima ácida 'Tahiti'

\begin{tabular}{lccccc}
\hline \multicolumn{1}{c}{ TRATAMENTOS } & \multicolumn{5}{c}{ Dias $^{\top}$} \\
\cline { 2 - 6 } & 0 & 15 & 30 & 45 & 60 \\
\hline Testemunha & 7,60 & $7,93 \mathrm{a}$ & $7,66 \mathrm{a}$ & $7,66 \mathrm{a}$ & $7,40 \mathrm{ab}$ \\
$100 \mathrm{mg} \cdot \mathrm{L}^{-1} \mathrm{GA}_{3}$ & 7,60 & $7,46 \mathrm{ab}$ & $7,06 \mathrm{ab}$ & $7,06 \mathrm{a}$ & $7,26 \mathrm{~b}$ \\
$200 \mathrm{mg} \cdot \mathrm{L}^{-1} \mathrm{GA}_{3}$ & 7,60 & $7,20 \mathrm{~b}$ & $6,86 \mathrm{~b}$ & $6,86 \mathrm{a}$ & $7,73 \mathrm{ab}$ \\
$400 \mathrm{mg} \cdot \mathrm{L}^{-1} \mathrm{GA}_{3}$ & 7,60 & $7,46 \mathrm{ab}$ & $6,86 \mathrm{~b}$ & $7,20 \mathrm{a}$ & $8,19 \mathrm{a}$ \\
$100 \mathrm{mg} \cdot \mathrm{L}^{-1} \mathrm{GA}_{3}+$ cera & 7,60 & $7,46 \mathrm{ab}$ & $7,13 \mathrm{ab}$ & $7,40 \mathrm{a}$ & $7,40 \mathrm{ab}$ \\
$200 \mathrm{mg} \cdot \mathrm{L}^{-1} \mathrm{GA}_{3}+$ cera & 7,60 & $7,33 \mathrm{ab}$ & $7,20 \mathrm{ab}$ & $6,83 \mathrm{a}$ & $7,46 \mathrm{ab}$ \\
$400 \mathrm{mg} \cdot \mathrm{L}^{-1} \mathrm{GA}_{3}+$ cera & 7,60 & $7,06 \mathrm{~b}$ & $6,93 \mathrm{ab}$ & $7,06 \mathrm{a}$ & $7,13 \mathrm{~b}$ \\
\hline C.V. $(\%)$ & - & 3,2 & 3,8 & 4,4 & 4,1 \\
\hline
\end{tabular}

1 Médias seguidas pela mesma letra não diferem significativamente pelo teste de Tukey a $5 \%$ de probabilidade.

TABELA 2 - Efeito da aplicação de ácido giberélico $\left(\mathrm{GA}_{3}\right)$ isolado ou associado com cera no pH do suco, durante o armazenamento refrigerado da lima ácida 'Tahiti'

\begin{tabular}{lccccc}
\hline TRATAMENTOS & \multicolumn{5}{c}{ Dias } \\
\cline { 2 - 6 } & 0 & 15 & 30 & 45 & 60 \\
\hline Testemunha & 2,50 & $2,54 \mathrm{a}$ & $2,58 \mathrm{~b}$ & $2,56 \mathrm{a}$ & $2,69 \mathrm{a}$ \\
$100 \mathrm{mg} \cdot \mathrm{L}^{-1} \mathrm{GA}_{3}$ & 2,50 & $2,50 \mathrm{a}$ & $2,60 \mathrm{ab}$ & $2,56 \mathrm{a}$ & $2,69 \mathrm{a}$ \\
$200 \mathrm{mg} \cdot \mathrm{L}^{-1} \mathrm{GA}_{3}$ & 2,50 & $2,54 \mathrm{a}$ & $2,62 \mathrm{a}$ & $2,57 \mathrm{a}$ & $2,69 \mathrm{a}$ \\
$400 \mathrm{mg} \cdot \mathrm{L}^{-1} \mathrm{GA}_{3}$ & 2,50 & $2,51 \mathrm{a}$ & $2,59 \mathrm{ab}$ & $2,56 \mathrm{a}$ & $2,71 \mathrm{a}$ \\
$100 \mathrm{mg} \cdot \mathrm{L}^{-1} \mathrm{GA}_{3}+$ cera & 2,50 & $2,55 \mathrm{a}$ & $2,61 \mathrm{ab}$ & $2,57 \mathrm{a}$ & $2,70 \mathrm{a}$ \\
$200 \mathrm{mg} \cdot \mathrm{L}^{-1} \mathrm{GA}_{3}+$ cera & 2,50 & $2,54 \mathrm{a}$ & $2,62 \mathrm{ab}$ & $2,55 \mathrm{a}$ & $2,71 \mathrm{a}$ \\
$400 \mathrm{mg} \cdot \mathrm{L}^{-1} \mathrm{GA}_{3}+$ cera & 2,50 & $2,56 \mathrm{a}$ & $2,62 \mathrm{ab}$ & $2,56 \mathrm{a}$ & $2,71 \mathrm{a}$ \\
\hline C.V. $(\%)$ & - & 1,2 & 0,6 & 0,7 & 0,7 \\
\hline
\end{tabular}

1 Médias seguidas pela mesma letra não diferem significativamente pelo teste de Tukey a $5 \%$ de probabilidade. 
BIASI, L. A.; ZANETTE, F. Ácido Giberélico Isolado...

TABELA 3 - Efeito da aplicação de ácido giberélico $\left(\mathrm{GA}_{3}\right)$ isolado ou associado com cera na acidez do suco, expressa em $\mathrm{ml}$ de $\mathrm{NaOH} 1 \mathrm{~N}$, durante o armazenamento refrigerado da lima ácida 'Tahiti'

\begin{tabular}{lcrrrr}
\hline TRATAMENTOS & \multicolumn{5}{c}{ Dias $^{1}$} \\
\cline { 2 - 6 } & 0 & 15 & 30 & 45 & 60 \\
\hline Testemunha & 10,16 & $10,53 \mathrm{a}$ & $10,23 \mathrm{a}$ & $9,60 \mathrm{a}$ & $8,63 \mathrm{a}$ \\
$100 \mathrm{mg} \cdot \mathrm{L}^{-1} \mathrm{GA}_{3}$ & 10,16 & $9,46 \mathrm{a}$ & $9,76 \mathrm{a}$ & $9,06 \mathrm{a}$ & $9,03 \mathrm{a}$ \\
$200 \mathrm{mg} \cdot \mathrm{L}^{-1} \mathrm{GA}_{3}$ & 10,16 & $9,33 \mathrm{a}$ & $9,63 \mathrm{a}$ & $8,46 \mathrm{a}$ & $9,10 \mathrm{a}$ \\
$400 \mathrm{mg} \cdot \mathrm{L}^{-1} \mathrm{GA}_{3}$ & 10,16 & $10,50 \mathrm{a}$ & $10,20 \mathrm{a}$ & $9,46 \mathrm{a}$ & $9,13 \mathrm{a}$ \\
$100 \mathrm{mg} \cdot \mathrm{L}^{-1} \mathrm{GA}_{3}+$ cera & 10,16 & $9,76 \mathrm{a}$ & $10,90 \mathrm{a}$ & $9,00 \mathrm{a}$ & $9,26 \mathrm{a}$ \\
$200 \mathrm{mg} \cdot \mathrm{L}^{-1} \mathrm{GA}_{3}+$ cera & 10,16 & $9,60 \mathrm{a}$ & $10,10 \mathrm{a}$ & $9,03 \mathrm{a}$ & $8,66 \mathrm{a}$ \\
$400 \mathrm{mg} \cdot \mathrm{L}^{-1} \mathrm{GA}_{3}+$ cera & 10,16 & $9,80 \mathrm{a}$ & $10,16 \mathrm{a}$ & $8,83 \mathrm{a}$ & $8,56 \mathrm{a}$ \\
\hline C.V. $\%$ (\%) & - & 5,9 & 5,2 & 7,0 & 4,5 \\
\hline
\end{tabular}

Médias seguidas pela mesma letra não diferem significativamente pelo teste de Tukey a $5 \%$ de probabilidade.

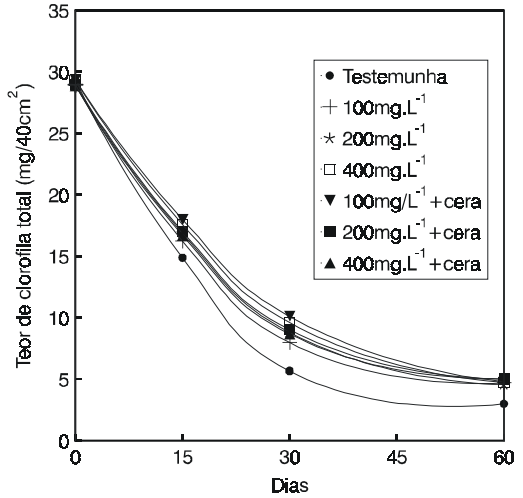

Testemunha: $Y=29,35-1,14 X+0,011 X^{2} ; R^{2}=0,99^{* * *}$ 100mg. $L^{-1}: Y=28,93-0,99 X+0,009 X^{2}: R^{2}=0,98^{\star * \star}$ 200mg. $L^{-1}: Y=28,9-0,93 X+0,008 X^{2}: R^{2}=0,98^{\star * \star}$ 400mg. $L^{-1}: Y=29,34-0,8 X+0,008 X^{2}: R^{2}=0,99 * * *$ 100mg. $L^{-1}+$ cera: $Y=29,36-0,87 X+0,007 X^{2}: R^{2}=0,99 * * *$ 200mg. $L^{-1}+$ cera: $Y=29,01-0,93 X+0,008 X^{2}: R^{2}=0,98^{\star \star}$ 400mg. $L^{-1}+$ cera: $Y=28,88-0,95 X+0,009 X^{2}: R^{2}=0,98^{\star *}$

FIGURA 1 - Teor de clorofila total da casca da lima ácida 'Tahiti' submetida a diferentes concentrações de ácido giberélico, com ou sem cera, durante 60 dias de armazenamento a $7^{\circ} \mathrm{C}$, dentro de sacos plásticos perfurados.

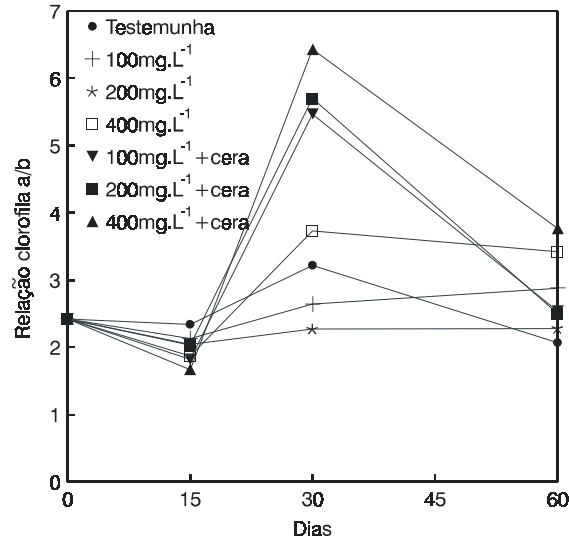

FIGURA 2 - Relação clorofila a/b da casca da lima ácida 'Tahiti' submetida a diferentes concentrações de ácido giberélico, com ou sem cera, durante 60 dias de armazenamento a $7^{\circ} \mathrm{C}$, dentro de sacos plásticos perfurados.

TABELA 4 - Efeito da aplicação de ácido giberélico $\left(\mathrm{GA}_{3}\right)$ isolado ou associado com cera no teor de clorofila total, em mg. $40 \mathrm{~cm}^{-2}$ de casca, dos frutos durante o armazenamento refrigerado da lima ácida 'Tahiti'

\begin{tabular}{lcccc}
\hline TRATAMENTOS & \multicolumn{5}{c}{ Dias $^{1}$} \\
\cline { 2 - 5 } & 0 & 15 & 30 & 60 \\
\hline Testemunha & 29,59 & $14,26 \mathrm{a}$ & $6,13 \mathrm{~b}$ & $2,91 \mathrm{~b}$ \\
$100 \mathrm{mg} \cdot \mathrm{L}^{-1} \mathrm{GA}_{3}$ & 29,59 & $14,51 \mathrm{a}$ & $9,29 \mathrm{a}$ & $4,50 \mathrm{a}$ \\
$200 \mathrm{mg} \cdot \mathrm{L}^{-1} \mathrm{GA}_{3}$ & 29,59 & $15,06 \mathrm{a}$ & $10,19 \mathrm{a}$ & $4,36 \mathrm{ab}$ \\
$400 \mathrm{mg} \cdot \mathrm{L}^{-1} \mathrm{GA}_{3}$ & 29,59 & $16,94 \mathrm{a}$ & $10,10 \mathrm{a}$ & $4,80 \mathrm{a}$ \\
$100 \mathrm{mg} \cdot \mathrm{L}^{-1} \mathrm{GA}_{3}+$ cera & 29,59 & $17,39 \mathrm{a}$ & $10,56 \mathrm{a}$ & $4,74 \mathrm{a}$ \\
$200 \mathrm{mg} \cdot \mathrm{L}^{-1} \mathrm{GA}_{3}+$ cera & 29,59 & $15,48 \mathrm{a}$ & $10,20 \mathrm{a}$ & $4,83 \mathrm{a}$ \\
$400 \mathrm{mg} \cdot \mathrm{L}^{-1} \mathrm{GA}_{3}+$ cera & 29,59 & $14,84 \mathrm{a}$ & $10,09 \mathrm{a}$ & $4,80 \mathrm{a}$ \\
\hline C.V. $\%)$ & - & 16,1 & 6,6 & 12,4 \\
\hline
\end{tabular}

1 Médias seguidas pela mesma letra não diferem significativamente pelo teste de Tukey a $5 \%$ de probabilidade. 
Com o aumento do tempo de armazenamento, houve decréscimo no teor de clorofila total da casca das frutas, sendo esse comportamento semelhante em todos os tratamentos, mas com maior degradação na testemunha (Figura 1). A tendência observada em todos os tratamentos foi melhor representada pelas equações quadráticas de regressão, que apresentaram elevados coeficientes de determinação (Figura 1). A degradação da clorofila foi semelhante à observada nos trabalhos de Carvalho et al. (3) e Manfroi et al. (12), com redução bastante acentuada no início do armazenamento, atingindo cerca de 50\% nos primeiros 15 dias. Já aos 30 dias de conservação, a degradação foi bem menos acentuada para os tratamentos com $\mathrm{GA}_{3}$, permanecendo em torno de $33 \%$, enquanto na testemunha, a perda da clorofila foi de $57,1 \%$.

Quanto à relação entre as clorofilas a e b, foram verificadas diferenças significativas aos 15 e 30 dias. Com 15 dias de armazenamento, a relação na testemunha foi superior ao tratamento com $400 \mathrm{mg} \cdot \mathrm{L}^{-1}$ de GA3 + cera, mas não diferiu dos demais. Já aos 30 dias, houve uma inversão no comportamento, e o tratamento com $400 \mathrm{mg} . \mathrm{L}^{-1} \mathrm{de}$ GA3 + cera, que aos 15 dias foi inferior, apresentou a maior relação. Nesta data de avaliação, houve tendência de os tratamentos com utilização de cera apresentarem maior relação clorofila a/b (Tabela 5). De forma geral, houve tendência de aumento na relação entre a clorofila $a / b$ até 30 dias de armazenamento com posterior redução (Figura 2). Este comportamento também foi encontrado por Carvalho et al. (3), Manfroi et al. (12) e Jahn e Young (10), sendo atribuído ao fato de a clorofila b sofrer degradação inicial mais intensa, elevando a relação clorofila a/b, e posterior inversão com degradação maior da clorofila a.

TABELA 5 - Efeito da aplicação de ácido giberélico $\left(\mathrm{GA}_{3}\right)$ isolado ou associado com cera na relação clorofila a/b da casca dos frutos durante o armazenamento refrigerado da lima ácida 'Tahiti'

\begin{tabular}{lcccc}
\hline TRATAMENTOS & \multicolumn{3}{c}{ Dias $^{1}$} \\
\cline { 2 - 5 } & 0 & $15^{2}$ & $30^{2}$ & $60^{2}$ \\
\hline Testemunha & 2,42 & $2,34 \mathrm{a}$ & $3,22 \mathrm{ab}$ & $2,07 \mathrm{a}$ \\
$100 \mathrm{mg} \cdot \mathrm{L}^{-1} \mathrm{GA}_{3}$ & 2,42 & $2,13 \mathrm{ab}$ & $2,64 \mathrm{ab}$ & $2,88 \mathrm{a}$ \\
$200 \mathrm{mg} \cdot \mathrm{L}^{-1} \mathrm{GA}_{3}$ & 2,42 & $2,04 \mathrm{ab}$ & $2,27 \mathrm{~b}$ & $2,28 \mathrm{a}$ \\
$400 \mathrm{mg} \cdot \mathrm{L}^{-1} \mathrm{GA}_{3}$ & 2,42 & $1,87 \mathrm{ab}$ & $3,73 \mathrm{ab}$ & $3,42 \mathrm{a}$ \\
$100 \mathrm{mg} \cdot \mathrm{L}^{-1} \mathrm{GA}_{3}+$ cera & 2,42 & $1,82 \mathrm{ab}$ & $5,48 \mathrm{ab}$ & $2,54 \mathrm{a}$ \\
$200 \mathrm{mg} \cdot \mathrm{L}^{-1} \mathrm{GA}_{3}+$ cera & 2,42 & $2,03 \mathrm{ab}$ & $5,70 \mathrm{ab}$ & $2,50 \mathrm{a}$ \\
$400 \mathrm{mg} \cdot \mathrm{L}^{-1} \mathrm{GA}_{3}+$ cera & 2,42 & $1,67 \mathrm{~b}$ & $6,43 \mathrm{a}$ & $3,77 \mathrm{a}$ \\
\hline $\mathrm{C} . \mathrm{V} .(\%)$ & - & 6,3 & 18,1 & 19,6 \\
\hline
\end{tabular}

Médias seguidas pela mesma letra não diferem significativamente pelo teste de Tukey a $5 \%$ de probabilidade.

2 Dados transformados para análise em log $(x+1)^{1 / 2}$.

\section{CONCLUSÕES}

1) A aplicação de $\mathrm{GA}_{3}$ até a dose de $400 \mathrm{mg} \cdot \mathrm{L}^{-1}$, com ou sem a utilização de cera, não alterou a qualidade do suco da lima ácida 'Tahiti'.

2) A aplicação de $\mathrm{GA}_{3}$ em todas as doses testadas, independente da aplicação de cera, foi eficiente na manutenção da coloração verde da casca da lima ácida 'Tahiti', sendo este efeito mais pronunciado após 30 dias de armazenamento.
3) Após 60 dias de armazenamento, houve pequeno aumento do pH e redução na acidez do suco.

\section{AGRADECIMENTOS}

Os autores agradecem a ABBOTT Laboratórios do Brasil Ltda. pelo apoio para a realização deste experimento.

\section{REFERÊNCIAS}

1 ARNON, D.I. Cooper enzymes in isoled chloroplasts polyphenoloxidase in Beta vulgaris. Plant Physiology, Lancaster, v. 24, p. 1-15, 1949.

2 BARROS, S.A.; RODRIGUES, J.D.; RODRIGUES, S.D.; PEDRAS, J.F. Efeito do ácido giberélico e do uniconazole, na fisiologia pós-colheita do limão 'Tahiti' (Citrus latifolia Tanaka). Revista Brasileira de Fruticultura, Cruz das Almas, v. 13, n. 3, p. 223-226, 1991.

3 CARVALHO, R.I.N.; FIOVARANÇO, J.C.; MANFROI, V.; LUCCHESE, O.A.; BENDER, R.J. Efeito de embalagens plásticas 
BIASI, L. A.; ZANETTE, F. Ácido Giberélico Isolado...

na frigoconservação do limão "Tahiti" (Citrus latifolia Tanaka). Revista do Setor de Ciências Agrárias, Curitiba, v. 12, n. 1-2, p. 31-38, 1992/3.

4 CARVALHO, V.D.; NOGUEIRA, D.J.P. Qualidade, maturação e colheita dos citros. Informe Agropecuário, Belo Horizonte, v. 5 , n. 52 , p. 61-67, 1979.

5 COELHO, Y.S. Lima ácida 'Tahiti' para exportação: aspectos técnicos da produção. Brasília: EMBRAPA-SPI, 1993. 35p. (Série Publicações Técnicas FRUPEX, 1).

6 DONADIO, L.C.; FIGUEIREDO, J.O.; PIO, R.M. Variedades cítricas brasileiras. Jaboticabal: FUNEP, 1995. 228p.

7 ECHEVERRIA, E.; VALICH, J. Enzymes of sugar and acid metabolism in stored "Valencia" oranges. Journal of American Society for Horticultural Science, Alexandria, v. 114, n. 3, p. 445-449, 1989.

8 FIGUEIREDO, J.O. Variedades copa de valor comercial. In: RODRIGUES, O.; VIÉGAS, F.; POMPEU JUNIOR, J.; AMARO, A.A. Citricultura brasileira. 2 ed. Campinas: Fundação Cargill, 1991. p.228-264.

9 FIORAVANÇO, J.C.; MANICA, I.; PAIVA, M.C. Efeito da citocinina, cera e embalagem sobre algumas características bioquímicas do limão 'Tahiti' armazenado em temperatura controlada. Revista Brasileira de Fruticultura, Cruz das Almas, v. 15, n. 3, p. 33-42, 1993.

10 JAHN, O.L.; YOUNG, R. Changes in chlorophyl $a, b$ and the $a / b$ ratio during color development in citrus fruit. Journal of American Society for Horticultural Science, Alexandria, v. 101, n. 4, p. 416-418, 1976.

11 LUDFORD, P.M. Postharvest hormone changes in vegetables and fruit. In: DAVIES, PJ. Plant hormones. 2 ed. Dordrecht: Kluwer Academic Publishers. 1995. p.725-750.

12 MANFROI, V.; LUCCHESE, O.A.; CARVALHO, R.I.N.; FIOVARANÇO, J.C.; BENDER, R.J. Efeito do 2,4-D na frigoconservação de limão Tahiti (Citrus latifolia Tanaka). B. CEPPA, Curitiba, v. 14, n. 1, p. 77-88, 1996.

13 PURVIS, A.C. Effects of film thickness and storage temperature on water loss and internal quality of seal-packaged grapefruit. Journal of American Society for Horticultural Science, Alexandria, v. 108, n. 4, p. 562-566, $1983 a$.

14 PURVIS, A.C. Moisture loss and juice quality from waxed and individual seal-packaged citrus fruits. Proceedings of Florida State Horticultural Society, Tallahassee, v. 96, p. 327-329, 1983b. 